\title{
Overview: Age-Hardenable Microalloying in Magnesium
}

\author{
JAMES E. SAAL ${ }^{1,3,4}$ and DMITRY ORLOV ${ }^{2,5}$ \\ 1.-QuesTek Innovations LLC, Evanston, IL 60201, USA. 2.-Division of Materials Engineering, \\ Department of Mechanical Engineering, LTH, Lund University, 22363 Lund, Sweden. 3.-e-mail: \\ jsaal531@gmail.com. 4.—e-mail: jsaal@questek.com. 5.—e-mail: dmytro.orlov@material.lth.se
}

As light weighting of vehicles becomes an urgent way to achieve increasingly stringent environmental standards, the introduction of low-density and high-abundance materials is becoming more important. Among these, magnesium (Mg) alloys are prime candidates for structural applications. However, critical issues with strength and ductility remain a roadblock for structural $\mathrm{Mg}$ alloys relative to $\mathrm{Al}$ alloys and steels. New designs of $\mathrm{Mg}$ alloys are needed to achieve in full the potential benefits lightweight magnesium can provide. These designs must necessarily use novel mechanisms of strength and ductility control to improve fracture resistance and formability over current alloys if complex system-critical Mg components are to be manufactured at scale and at cost.

A primary method for improving strength in cast and wrought $\mathrm{Mg}$ alloys is age hardening. Upon quenching a supersaturated solid solution of alloyed $\mathrm{Mg}$ below the single-phase hexagonal close-packed (hcp) solvus, precipitation of secondary phase(s) occurs during aging. Typically, a series of phase transformations takes place and produces a procession of metastable secondary phases that vary in composition, crystal structure, and morphology. Because alloy hardness depends on microstructure, it also evolves with aging temperature and time; it achieves maxima due to a particular precipitate structure.

During the past 10 years, significant advances have been made in our mechanistic understanding of structure-property relationships in $\mathrm{Mg}$ alloys. They have been fueled primarily by novel microscopy techniques and theoretical computations. Precipitation sequences of many $\mathrm{Mg}$ alloys have been evaluated in great experimental detail, revealing specific strengthening mechanisms for specific alloys. ${ }^{1}$ For instance, during aging of $\mathrm{Mg}$ alloys containing light rare-earth elements, $\beta^{\prime \prime}$

\footnotetext{
James E. Saal and Dmitry Orlov are the guest editors for the Magnesium Committee of the TMS Light Metals Division (LMD); and coordinators of the topic Age-Hardenable Microalloying in Magnesium
}

plate-type particles tend to precipitate on prismatic planes of the hcplattice, preventing basal slip and thus improving strength. Theoretical ab initio calculations have discovered that the cause of this particular prismatic precipitate morphology is a specific balance of anisotropies in the chemical interfacial energy and the elastic coherency strain energy. ${ }^{2}$ Currently, efforts are underway to search for less costly elements that can produce similar precipitates using this mechanistic model. ${ }^{3}$

An understanding of strengthening mechanisms in existing $\mathrm{Mg}$ alloys is necessary for the development of novel alloys. Alloy design fundamentally depends on such processing-structure-property relationships (e.g., aging $\rightarrow$ precipitate morphology $\rightarrow$ strength). Thus, the development of a competitive $\mathrm{Mg}$ alloy for significant light weighting in vehicles requires exploration of fundamental strength and ductility mechanisms. To that end, the following articles being published under the topic of Age-Hardenable Microalloying in $\mathrm{Mg}$ provide excellent details and research on the subject. To download any of the papers, follow the URL http:// link.springer.com/journal/11837/67/10/page/1 to the table of contents page for the October 2015 issue (vol. 67, no. 10).

The first article by Mendis, Kainer, and Hort is titled, "High-Strength Magnesium Alloys Through Precipitation Hardening and Microalloying: Considerations for Alloy Design."It reviews recent advances in $\mathrm{Mg}$ strengthening through microalloying and summarizes design strategies that should be explored to achieve the properties necessary for the broad commercial utilization of $\mathrm{Mg}$. Three primary microstructural features are identified as degrees of freedom upon which alloying can affect the hardness of base $\mathrm{Mg}$. These are grain boundaries, matrix composition and structure, and precipitates. A critical assessment of the current state of research into these pathways for alloying culminates in a primary criterion for enhancing the agehardening response by alloying: The formation of a large number of heterogeneous nucleation sites to 
which fast-diffusing solute additions can migrate and form strengthening precipitates. This design strategy provides a clear roadmap for future work in the field outlining materials data and models necessary to verify, explore, and exploit suggested mechanisms.

In the second article titled, "Solid-Solution Hardening in Mg-Gd-TM (TM = Ag, Zn, and Zr) Alloys: An Integrated Density Functional Theory and Electron Work Function Study," William Yi Wang, Shun Li Shang, Yi Wang, Hongyeun Kim, Kristopher A. Darling, Laszlo J. Kecskes, Suveen N. Mathaudhu, Xi Dong Hui, and Zi-Kui Liu employ theoretical modeling approaches to elaborate on strengthening in rare-earth-containing $\mathrm{Mg}$ alloys with long period stacking ordered (LPSO) structures. The authors suggest two primary considerations for LPSO formation and strengthening: the formation of face-centered cubic (fcc)-type stacking faults in $\mathrm{Mg}$ hcplattice, and chemical ordering of the rare-earth atoms along the faults. The authors of this paper have applied an electron work function to directly correlate these effects to the observed hardness response in Gd-containing $\mathrm{Mg}$ alloys. Such fundamental insight into causal precipitate-hardness structure-property relationships is critical in determining novel microalloying techniques leading to further advancements in $\mathrm{Mg}$ alloys.

The last paper by Zhikun Qu, Pengfei Fei, and Fuizhi Wuis titled, "Microstructure Evolution and Hardness Variation of Mg-9Li-6Al- $x \mathrm{La}(x=0$ and
2.0) Alloys Under Different Aging Parameters." It focuses on the microstructural characterization of a $\mathrm{Li}-, \mathrm{Al}-$, and La-containing $\mathrm{Mg}$ alloys and reveals a correlation between the evolution of microstructure and changes in hardness. Namely, morphology of the $\mathrm{Li}_{2} \mathrm{MgAl} \theta$-phase evolves with aging, which leads to a steady decrease in hardness, while precipitation of $\mathrm{Al} \mathrm{Li}$ at higher aging temperatures prevents such a decrease, causing a flat hardness response with time. The addition of La appears also to reduce the decrease in hardness with aging due to the formation of $\mathrm{Al}-\mathrm{La}$ compounds. It is precisely such mechanistic processing-structure-property evidence that is necessary for the development of microalloying concepts in $\mathrm{Mg}$ alloy design, which capitalizes on the wealth of precipitate characterization by advanced techniques. ${ }^{1}$

These articles provide a snapshot of microalloying-based approaches aimed at overcoming the challenges in practical application of $\mathrm{Mg}$ alloys for structural components. They convincingly demonstrate that design of competitive $\mathrm{Mg}$ alloys necessarily requires integration of advanced structureproperty characterization techniques with theoretical models.

\section{REFERENCES}

1. J.-F. Nie, Metall. Mater. Trans. A 43, 3891 (2012).

2. A. Issa, J.E. Saal, and C. Wolverton, Acta Mater. 65, 240 (2014).

3. A. Issa, J.E. Saal, and C. Wolverton, Acta Mater. 83, 75 (2015). doi:10.1016/j.actamat.2014.09.024. 